Supplement of Hydrol. Earth Syst. Sci., 23, 1611-1631, 2019

https://doi.org/10.5194/hess-23-1611-2019-supplement

(c) Author(s) 2019. This work is distributed under

the Creative Commons Attribution 4.0 License.

(c) (1)
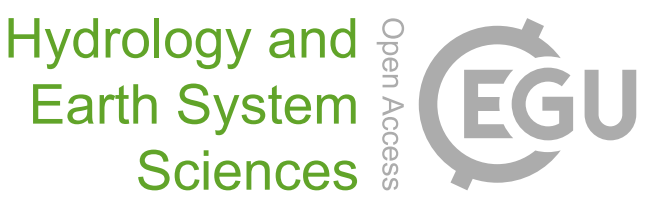

Supplement of

\title{
Projected decrease in wintertime bearing capacity on different forest and soil types in Finland under a warming climate
}

Ilari Lehtonen et al.

Correspondence to: Ilari Lehtonen (ilari.lehtonen@fmi.fi)

The copyright of individual parts of the supplement might differ from the CC BY 4.0 License. 
Table S1. The calibrated parameter values with standard deviations at 10 and $20 \mathrm{~cm}$ depths as averaged over all the validation stations (excluding the peat stations having no soil temperature observations from these depth) after the first calibration round.

\begin{tabular}{|l|l|l|}
\hline Parameter & \multicolumn{2}{l|}{ Calibrated values with standard deviations } \\
\cline { 2 - 3 } & $10 \mathrm{~cm}$ depth & $20 \mathrm{~cm}$ depth \\
\hline Soil thermal conductivity, $K_{T}\left(\mathrm{~W} \mathrm{~m}^{-1} \mathrm{~K}^{-1}\right)$ & $0.13 \pm 0.06$ & $0.45 \pm 0.25$ \\
\hline Specific heat capacity of soil, $C_{S}\left(10^{6} \mathrm{~J} \mathrm{~m}^{-3} \mathrm{~K}^{-1}\right)$ & $1.40 \pm 0.24$ & $1.78 \pm 0.56$ \\
\hline Specific heat capacity due to freezing and thawing, $C_{I C E}\left(\mathrm{~J} \mathrm{~m}^{-3} \mathrm{~K}^{-1}\right)$ & $10.9 \pm 1.7$ & $11.2 \pm 2.6$ \\
\hline Empirical snow parameter, $f_{S}\left(\mathrm{~m}^{-1}\right)$ & $7.6 \pm 3.9$ & $8.5 \pm 2.1$ \\
\hline Lower soil thermal conductivity, $K_{T, L O W}\left(\mathrm{~W} \mathrm{~m}^{-1} \mathrm{~K}^{-1}\right)$ & $0.74 \pm 0.19$ & $0.63 \pm 0.32$ \\
\hline Lower soil specific heat capacity, $C_{S, L O W}\left(\mathrm{~J} \mathrm{~m}^{-3} \mathrm{~K}^{-1}\right)$ & $1.89 \pm 0.62$ & $1.39 \pm 0.60$ \\
\hline Lower soil temperature depth, $Z_{l}(\mathrm{~m})$ & $8.4 \pm 4.0$ & $5.6 \pm 3.3$ \\
\hline
\end{tabular}


Table S2. The coefficients of determination $\left(R^{2}\right)$ for modelled soil temperatures for different soil types at different depths when using in the model calculations the calibrated parameters listed in Table 3. The highest $R^{2}$ for each station and measurement depth is shown in bold.

\begin{tabular}{|c|c|c|c|c|c|c|c|c|c|c|c|c|}
\hline Station name & Soil type & $5 \mathrm{~cm}$ & $10 \mathrm{~cm}$ & $15 \mathrm{~cm}$ & $20 \mathrm{~cm}$ & $30 \mathrm{~cm}$ & $40 \mathrm{~cm}$ & $50 \mathrm{~cm}$ & $70 \mathrm{~cm}$ & $\begin{array}{l}100 \\
\mathrm{~cm}\end{array}$ & $\begin{array}{l}150 \\
\mathrm{~cm}\end{array}$ & $\begin{array}{l}200 \\
\mathrm{~cm}\end{array}$ \\
\hline Lettosuo & $\begin{array}{l}\text { Clay/silt } \\
\text { Sand } \\
\text { Peat }\end{array}$ & $\begin{array}{l}0.93 \\
0.88 \\
\mathbf{0 . 9 6}\end{array}$ & & $\begin{array}{l}0.90 \\
0.85 \\
\mathbf{0 . 9 7}\end{array}$ & & $\begin{array}{l}0.86 \\
0.80 \\
\mathbf{0 . 9 8}\end{array}$ & $\begin{array}{l}0.81 \\
0.72 \\
\mathbf{0 . 9 7}\end{array}$ & & & & & \\
\hline Anjala & $\begin{array}{l}\text { Clay/silt } \\
\text { Sand } \\
\text { Peat }\end{array}$ & & $\begin{array}{l}\mathbf{0 . 9 7} \\
0.95 \\
0.95\end{array}$ & & $\begin{array}{l}\mathbf{0 . 9 8} \\
0.97 \\
0.93\end{array}$ & $\begin{array}{l}\mathbf{0 . 9 8} \\
0.98 \\
0.88\end{array}$ & & $\begin{array}{l}\mathbf{0 . 9 8} \\
0.97 \\
0.79\end{array}$ & $\begin{array}{l}\mathbf{0 . 9 9} \\
0.97\end{array}$ & $\begin{array}{l}\mathbf{0 . 9 8} \\
0.94\end{array}$ & $\begin{array}{l}\mathbf{0 . 9 4} \\
0.91\end{array}$ & $\begin{array}{l}\mathbf{0 . 8 7} \\
0.83\end{array}$ \\
\hline Jyväskylä & $\begin{array}{l}\text { Clay/silt } \\
\text { Sand } \\
\text { Peat }\end{array}$ & & $\begin{array}{l}0.94 \\
0.90 \\
\mathbf{0 . 9 8}\end{array}$ & & $\begin{array}{l}\mathbf{0 . 9 7} \\
0.93 \\
0.97\end{array}$ & $\begin{array}{l}\mathbf{0 . 9 8} \\
0.94 \\
0.94\end{array}$ & & & $\begin{array}{l}\mathbf{0 . 9 8} \\
0.94\end{array}$ & $\begin{array}{l}\mathbf{0 . 9 8} \\
0.93\end{array}$ & $\begin{array}{l}\mathbf{0 . 9 5} \\
0.91\end{array}$ & $\begin{array}{l}\mathbf{0 . 9 1} \\
0.89\end{array}$ \\
\hline Ylistaro & $\begin{array}{l}\text { Clay/silt } \\
\text { Sand } \\
\text { Peat }\end{array}$ & & & & $\begin{array}{l}\mathbf{0 . 9 7} \\
0.96 \\
0.94\end{array}$ & & & $\begin{array}{l}0.97 \\
\mathbf{0 . 9 8} \\
0.74\end{array}$ & & $\begin{array}{l}\mathbf{0 . 9 7} \\
0.94\end{array}$ & & $\begin{array}{l}\mathbf{0 . 8 9} \\
0.87\end{array}$ \\
\hline Maaninka & $\begin{array}{l}\text { Clay/silt } \\
\text { Sand } \\
\text { Peat }\end{array}$ & & $\begin{array}{l}\mathbf{0 . 9 7} \\
0.94 \\
0.93\end{array}$ & & $\begin{array}{l}\mathbf{0 . 9 8} \\
0.97 \\
0.90\end{array}$ & $\begin{array}{l}0.98 \\
\mathbf{0 . 9 8} \\
0.85\end{array}$ & & $\begin{array}{l}0.98 \\
\mathbf{0 . 9 9} \\
0.74\end{array}$ & $\begin{array}{l}\mathbf{0 . 9 8} \\
0.98\end{array}$ & $\begin{array}{l}\mathbf{0 . 9 8} \\
0.97\end{array}$ & $\begin{array}{l}\mathbf{0 . 9 2} \\
0.92\end{array}$ & $\begin{array}{l}\mathbf{0 . 8 3} \\
0.81\end{array}$ \\
\hline Apukka & $\begin{array}{l}\text { Clay/silt } \\
\text { Sand } \\
\text { Peat }\end{array}$ & & $\begin{array}{l}\mathbf{0 . 9 6} \\
0.92 \\
0.94\end{array}$ & & $\begin{array}{l}\mathbf{0 . 9 8} \\
0.95 \\
0.92\end{array}$ & $\begin{array}{l}\mathbf{0 . 9 7} \\
0.97 \\
0.88\end{array}$ & & $\begin{array}{l}0.96 \\
0.97 \\
0.78\end{array}$ & $\begin{array}{l}0.96 \\
\mathbf{0 . 9 7}\end{array}$ & $\begin{array}{l}\mathbf{0 . 9 7} \\
0.96\end{array}$ & $\begin{array}{l}0.93 \\
\mathbf{0 . 9 5}\end{array}$ & $\begin{array}{l}0.86 \\
0.91\end{array}$ \\
\hline Sodankylä & $\begin{array}{l}\text { Clay/silt } \\
\text { Sand } \\
\text { Peat }\end{array}$ & & $\begin{array}{l}0.94 \\
\mathbf{0 . 9 5} \\
0.90\end{array}$ & & $\begin{array}{l}0.95 \\
\mathbf{0 . 9 6} \\
0.89\end{array}$ & $\begin{array}{l}0.95 \\
0.97 \\
0.84\end{array}$ & & $\begin{array}{l}0.97 \\
0.98 \\
0.73\end{array}$ & $\begin{array}{l}0.97 \\
\mathbf{0 . 9 8}\end{array}$ & $\begin{array}{l}0.94 \\
\mathbf{0 . 9 8}\end{array}$ & $\begin{array}{l}0.87 \\
\mathbf{0 . 9 5}\end{array}$ & $\begin{array}{l}0.81 \\
\mathbf{0 . 8 9}\end{array}$ \\
\hline Lompolojänkkä & $\begin{array}{l}\text { Clay/silt } \\
\text { Sand } \\
\text { Peat }\end{array}$ & $\begin{array}{l}\mathbf{0 . 9 1} \\
0.91 \\
0.89\end{array}$ & & $\begin{array}{l}0.93 \\
0.93 \\
\mathbf{0 . 9 5}\end{array}$ & & $\begin{array}{l}0.87 \\
0.82 \\
\mathbf{0 . 9 2}\end{array}$ & & & & & & \\
\hline Kaamanen & $\begin{array}{l}\text { Clay/silt } \\
\text { Sand } \\
\text { Peat }\end{array}$ & $\begin{array}{l}0.85 \\
0.82 \\
\mathbf{0 . 8 5}\end{array}$ & & $\begin{array}{l}0.51 \\
0.50 \\
\mathbf{0 . 5 5}\end{array}$ & & $\begin{array}{l}0.96 \\
0.97 \\
0.83\end{array}$ & & & & & & \\
\hline Kevo & $\begin{array}{l}\text { Clay/silt } \\
\text { Sand } \\
\text { Peat }\end{array}$ & & $\begin{array}{l}0.88 \\
0.87 \\
\mathbf{0 . 8 9}\end{array}$ & & $\begin{array}{l}\mathbf{0 . 9 0} \\
0.88 \\
0.86\end{array}$ & & & $\begin{array}{l}0.92 \\
\mathbf{0 . 9 2} \\
0.75\end{array}$ & & $\begin{array}{l}0.87 \\
\mathbf{0 . 8 8}\end{array}$ & & $\begin{array}{l}0.66 \\
\mathbf{0 . 6 6}\end{array}$ \\
\hline
\end{tabular}


Table S3. The coefficients of determination $\left(R^{2}\right)$ for modelled snow depths at different stations for the validation periods 1962-1980 and 1981-2005 (the second and third column from the left) and for the calibration period 2006-2014 (the fourth column from the left). The three columns in the right show the modelled snow depth compared to the observed snow depth in percents averaged over the periods 1962-1980, 1981-2005 and 2006-2014.

\begin{tabular}{|l|l|l|l|l|l|l|}
\hline Station name & $\begin{array}{l}\mathrm{R}^{2} \\
1962-1980\end{array}$ & $\begin{array}{l}\mathrm{R}^{2} \\
1981-2005\end{array}$ & $\begin{array}{l}\mathrm{R}^{2} \\
2006-2014\end{array}$ & $\begin{array}{l}\text { \% of obs } \\
1962-1980\end{array}$ & $\begin{array}{l}\text { \% of obs } \\
1981-2005\end{array}$ & $\begin{array}{l}\text { \% obs } \\
2006-2014\end{array}$ \\
\hline Anjala & 0.91 & 0.89 & 0.96 & 71 & 95 & 103 \\
\hline Jyväskylä & 0.87 & 0.91 & 0.96 & 80 & 94 & 108 \\
\hline Ylistaro & 0.80 & 0.87 & 0.96 & 50 & 68 & 83 \\
\hline Maaninka & 0.79 & 0.92 & 0.96 & 60 & 84 & 96 \\
\hline Apukka & 0.95 & 0.92 & 0.84 & 75 & 96 & 131 \\
\hline Sodankylä & 0.91 & 0.93 & 0.95 & 51 & 65 & 85 \\
\hline Kevo & 0.83 & 0.89 & 0.94 & 54 & 83 & 73 \\
\hline
\end{tabular}

Table S4. Global climate model (GCM) simulations used in this study. More information about the models can be found in Flato et al. (2013).

\begin{tabular}{|c|c|c|c|}
\hline Model & Institution & $\begin{array}{l}\text { Resolution } \\
(\text { Lon } \times \text { Lat Levels) }\end{array}$ & Ensemble member \\
\hline CanESM2 & $\begin{array}{l}\text { Canadian Centre for Climate Modelling and } \\
\text { Analysis, Canada }\end{array}$ & $128 \times 64 \mathrm{~L} 35$ & r1i1p1 \\
\hline CNRM-CM5 & $\begin{array}{l}\text { National Centre for Meteorological Research, } \\
\text { Meteo-France, France }\end{array}$ & $256 \times 128 \mathrm{~L} 31$ & r1i1p1 \\
\hline GFDL-CM3 & $\begin{array}{l}\text { Geophysical Fluid Dynamics Laboratory, } \\
\text { USA }\end{array}$ & $144 \times 90 \mathrm{~L} 48$ & $\begin{array}{l}\text { r1i1p1 for RCP8.5 } \\
\text { r3i1p1 for RCP4.5 }\end{array}$ \\
\hline HadGEM2-ES & Met Office Hadley Centre, UK & $192 \times 145 \mathrm{~L} 38$ & r1i1p1 \\
\hline MIROC5 & $\begin{array}{l}\text { Atmosphere and Ocean Research Institute, } \\
\text { National Institute for Environmental Studies } \\
\text { and Japan Agency for Marine-Earth Science } \\
\text { and Technology, Japan }\end{array}$ & $256 \times 128 \mathrm{~L} 40$ & r2i1p1 \\
\hline MPI-ESM-MR & $\begin{array}{l}\text { Max Planck Institute for Meteorology, } \\
\text { Germany }\end{array}$ & $192 \times 96$ L95 & r1i1p1 \\
\hline
\end{tabular}


Table S5. Bias-adjusted regional climate model (RCM) simulations used in this study. More information about the models can be found in Jacob et al. (2014).

\begin{tabular}{|l|l|l|l|l|}
\hline Global model & Regional model & Bias adjustment name & CORDEX domain & Ensemble member \\
\hline CNRM-CM5 & CNRM-ARPEGE51 & IPSL-CDFT22 & EUR-11 & r1i1p1 \\
\hline CNRM-CM5 & SMHI-RCA4 & IPSL-CDFT22 & EUR-11 & r1i1p1 \\
\hline EC-EARTH & DMI-HIRHAM5 & IPSL-CDFT22 & EUR-11 & r3i1p1 \\
\hline EC-EARTH & KNMI-RACMO22E & IPSL-CDFT22 & EUR-11 & r1i1p1 \\
\hline EC-EARTH & SMHI-RCA4 & IPSL-CDFT22 & EUR-11 & r12i1p1 \\
\hline HadGEM2-ES & KNMI-RACMO22E & IPSL-CDFT22 & EUR-11 & r1i1p1 \\
\hline HadGEM2-ES & SMHI-RCA4 & IPSL-CDFT22 & EUR-11 & r1i1p1 \\
\hline IPSL-CM5A-MR & IPSL-INERIS-WRF331F & IPSL-CDFT22 & EUR-11 & r1i1p1 \\
\hline IPSL-CM5A-MR & SMHI-RCA4 & IPSL-CDFT22 & EUR-11 & r1i1p1 \\
\hline MPI-ESM-LR & MPI-CSC-REMO2009 & IPSL-CDFT22 & EUR-11 & r1i1p1 \\
\hline MPI-ESM-LR & SMHI-RCA4 & IPSL-CDFT22 & EUR-11 & r1i1p1 \\
\hline
\end{tabular}

